\title{
Synthesis and Electrical Properties of $\mathrm{TiO}_{2}$ Nanoparticles Embedded in Polyamide-6 Nanofibers Via Electrospinning
}

\author{
R. Nirmala ${ }^{1}$, Jin Won Jeong ${ }^{2,3}$, R. Navamathavan ${ }^{4}$, Hak Yong $\mathrm{Kim}^{2,3, *}$
}

(Received 17 March 2011; accepted 3 May 2011; published online 13 May 2011.)

\begin{abstract}
We report on the synthesis and characterizations of $\mathrm{TiO}_{2}$ nanoparticles embedded in polyamide- 6 composite nanofibers by using electrospinning technique. The influence of substrate on the electrical characteristics of polyamide- $6 / \mathrm{TiO}_{2}$ composite nanofibers was investigated. The resultant nanofibers exhibit good incorporation of $\mathrm{TiO}_{2}$ nanoparticles. The doping of $\mathrm{TiO}_{2}$ nanoparticles into the polyamide- 6 nanofibers were confirmed by high resolution transmission electron microscopy and energy dispersive X-ray spectroscopy. Photoluminescence (PL) and cathodoluminescence (CL) spectroscopy were also used to characterize the samples. The PL and CL spectra reveal that the as-spun polyamide-6/ $\mathrm{TiO}_{2}$ composite nanofibers consisted of overlapping of two broad emission bands due to the contribution of polyamide- 6 (centered at about $475 \mathrm{~nm}$ ), which might originate from organic functional groups of polyamide- 6 and $\mathrm{TiO}_{2}$ nanoparticles (centered around $550 \mathrm{~nm}$ ). The electrical conductivity of the polyamide- $6 / \mathrm{TiO}_{2}$ composite nanofibers on different substrates was carried out. It was found that the electrical conductivity of the polyamide- $6 / \mathrm{TiO}_{2}$ composite nanofibers on silicon substrate was in the range of $1 \sim 3 \mu \mathrm{A}$, and about 1 to $20 \mathrm{pA}$ for the paper and glass substrates.
\end{abstract}

Keywords: Electrospinning; Polyamide-6; Nanoparticles; Nanofibers; Electrical studies

Citation: R. Nirmala, Jin Won Jeong, R. Navamathavan and Hak Yong Kim, "Synthesis and Electrical Properties of $\mathrm{TiO}_{2}$ Nanoparticles Embedded in Polyamide-6 Nanofibers Via Electrospinning", Nano-Micro Lett. 3 (1), 56-61 (2011). http://dx.doi.org/10.3786/nml.v3i1.p56-61

In recent years, much attention has been paid to the preparation of one-dimensional (1D) nanomaterials, such as nanofibers, nanowires, nanorods, nanotubes, and nanobelts, which exhibit novel physical and chemical properties due to their unique characteristics for huge ratio of diameter to length, superior mechanical toughness, and so on [1-6]. Polymer based devices have attracted considerable attention in the fields of flexible functional and large-area electronics. Recently, many methods have been utilized to prepare 1D nanomaterials which includes chemical or physical vapor deposition, arc discharge, laser ablation, vapor- phase transport process, and a template-based method [7-12]. Among the various methods, electrospinning is simple, effective, and cost-effective approach for generating long fibers with diameters ranging from tens of nanometers up to micrometers. In the fabrication of polymer electronics, recent research has intensively focused on the electrospinning method as a unique way of synthesizing polymer nanofibers $[13,14]$.

Nanofiber structures are investigated for their electrical properties and device characterizations. The electrical conductance is a fundamental property encountered in every study of electrical transport phenom-

\footnotetext{
${ }^{1}$ Bio-nano System Engineering, Chonbuk National University, Jeonju, 561-756, South Korea

${ }^{2}$ Department of Organic Materials and Fiber Engineering, Chonbuk National University, Jeonju, 561-756, South Korea

${ }^{3}$ Center for Healthcare Technology \& Development, Chonbuk National University, Jeonju, 561-756, South Korea

${ }^{4}$ School of Advanced Materials Engineering, Chonbuk National University, Jeonju 561-756, South Korea

*Corresponding author. Tel.: +82-63-270-2351; fax: +82-63-270-4249. E-mail: khy@jbnu.ac.kr (HakYong Kim).
} 
ena in nanostructured materials. Polymer nanofibers can be more readily applicable if their electrical conductivities were enhanced. A semiconducting polymer, such as polyamide-6, decorated with metallic conducting nanoparticles furnishes an interesting system with the prospect of designing device functionality directly into the material. The electrical conductivities of polyamide- 6 nanofibers can be significantly improved by adding metal nanoparticles with high conductivities. The electrical property is known to influence the characteristics of the device and its stability under an electric field. Therefore, research activities have thus been intensified on tailoring the electrical property of nonconducting polymer composites by adding with electrically active materials. In our previous report, we have investigated the electrical properties of polyamide6/chitosan blended nanofibers [15]. Polymers and their composites have been used as sensing materials to detect targeted gases based on various sensing techniques $[16,17]$. However, the electrical properties of these polyamide- $6 / \mathrm{TiO}_{2}$ composite nanofibers have not been reported so far. Therefore, we have carried out the detailed electrical characterization of these composite polyamide- 6 nanofibers.

In this study, the effect of substrate type on the electrical properties of embedding of $\mathrm{TiO}_{2}$ nanoparticles in polyamide- 6 nanofibers has been investigated. We analyzed detailed electrical characteristics of these composite nanofibers as-spun on various substrates such as $\mathrm{Si}$, paper and glass. The morphological, luminescence and electrical characteristics of the as-spun polyamide- 6 composite nanofibers were investigated.

Polyamide-6 (KN120 grade, Kolon Industries, South Korea) and $\mathrm{TiO}_{2}$ nanoparticles (Aeroxide P25, $80 \%$ anatase $20 \%$ rutile, average particle size of $21 \mathrm{~nm}$ and specific surface area of $50 \pm 15 \mathrm{~m}^{2} \mathrm{~g}^{-1}$, Sigma-Aldrich Inc.) were used in making the polymer solution. The nanofibers were produced by dissolving polyamide- 6 pellets in $85 \%$ formic acid (analytical grade, Showa, Japan). After that the polymer solution was loaded into a $5 \mathrm{ml}$ plastic syringe equipped with a polystyrene micro-tip (0.3 $\mathrm{mm}$ inner diameter and $10 \mathrm{~mm}$ length), which was connected with a high-voltage power supply (CPS-60 K02V1, Chungpa EMT, South Korea). Electrospinning was performed at a voltage of about $22 \mathrm{kV}$. A grounded iron drum was rotated at a constant speed by a DC motor to collect the developing nanofibers on the silicon, paper and glass substrates, which were kept at a distance of $15 \mathrm{~cm}$ from the micro-tip. All experiments were conducted at room temperature.

The phase and crystallinity of $\mathrm{TiO}_{2}$ nanoparticles were characterized using a Rigaku X-ray diffractometer (XRD, Rigaku Co., Japan) with $\mathrm{Cu} \mathrm{K} \alpha(\lambda=1.54056$ $\AA)$ radiation over a range of $2 \theta$ angles from $20^{\circ}$ to $80^{\circ}$. The morphology of the as-spun polyamide- 6 nanofibers was observed by using scanning electron microscopy
(SEM, Hitachi S-7400, Hitachi, Japan) and high resolution transmission electron microscopy (HR-TEM, JEM-2010, JEOL, Japan). To confirm the embedding of nanoparticles, the chemical composition of the as-spun nanofiber mats were analyzed by energy dispersive X-ray (EDX) spectrometer attached with HRTEM. The optical property of polyamide- $6 / \mathrm{TiO}_{2}$ composite nanofibers was characterized by photoluminescence (PL) analysis using a $325 \mathrm{~nm}$ He-Cd laser and a cathodoluminescence (CL) attached to the field emission scanning electron microscopy (FE-SEM) system. Then the current-voltage (I-V) characteristic was measured for the ultrafine polyamide- 6 nanofibers by using a semiconductor parameter analyzer (4200-SCS, Keithley). I-V characteristic measurement was performed: a bundle of nanofibers (with thickness of approximately few $\mathrm{mm}$ and a length of few $\mu \mathrm{m}$ ) composed of polyamide- $6 / \mathrm{TiO}_{2}$ was glued to metal contacts. The electrode was made by using silver paste glue which ensured a perfect electrical contact between the metal and the fibers. In the experiments an increasing DC voltage was applied from -20 to $20 \mathrm{~V}$ to the metal contacts. The current flowing through the bundle was measured as a function of the applied voltage.

Figure 1 shows XRD pattern of the $\mathrm{TiO}_{2}$ nanoparticles. The anatase reflections are dominating in the reflection patterns and rutile is also present. As shown in the XRD data, the results affirm formation of the anatase, the existence of strong diffraction peaks at $2 \theta$ values of $25.25^{\circ}, 37.80^{\circ}, 38.50^{\circ}, 48.05^{\circ}, 53.9^{\circ}, 55.05^{\circ}$, $62.65^{\circ}, 68.85^{\circ}, 70.30^{\circ}, 75.05^{\circ}$ and $76.10^{\circ}$ corresponding to the crystal planes (101), (004), (112), (200), (105), (211), (204), (116), (220), (215) and (301), respectively, indicating the formation of the anatase (JCPDS card no 21-1272). And diffraction peaks at $2 \theta$ values of $36.00^{\circ}$ can be well indexed to (101) reflection of rutile phase (JCPDS card no 21-1276). The average crystal size is estimated by the Scherrer's formula [18]. The average crystal sizes are calculated to be of $20 \mathrm{~nm}$.

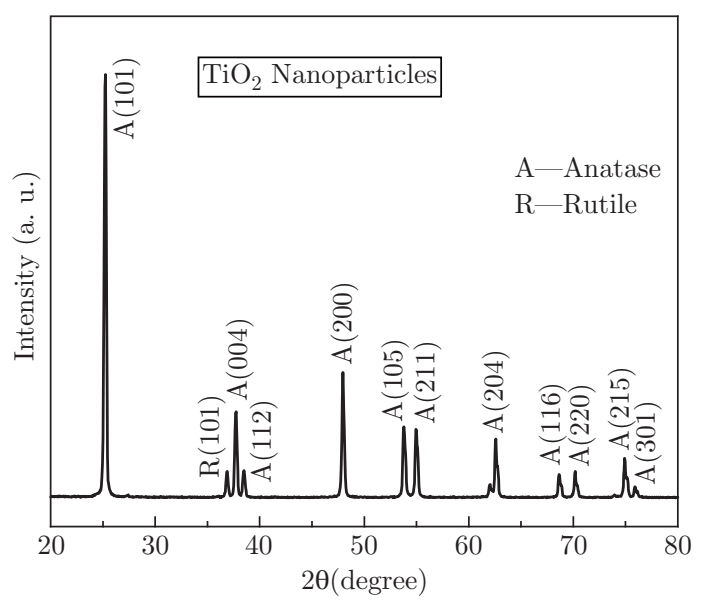

Fig. 1 XRD pattern of the $\mathrm{TiO}_{2}$ nanoparticles. 
Figure 2 shows the typical SEM image of polyamide$6 / \mathrm{TiO}_{2}$ composite nanofibers. This image shows the composite nanofibers exhibited smooth surface and bead-free uniform diameters along their lengths. On the other hand, the incorporation of $\mathrm{TiO}_{2}$ nanoparticles can be seen in the SEM images as shown in Fig. 2. The resultant composite nanofibers diameters were observed to be in the range of 100 to $150 \mathrm{~nm}$.

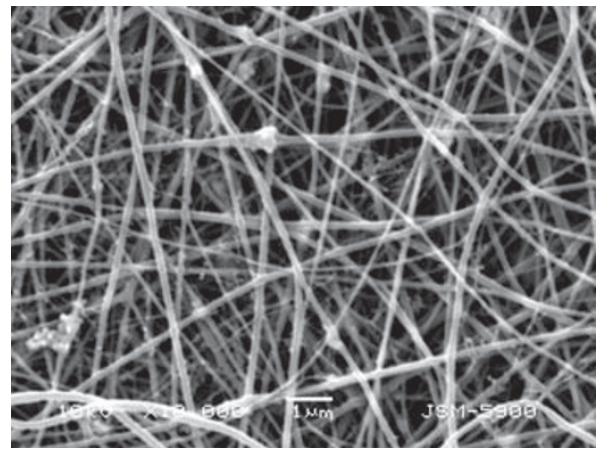

Fig. 2 SEM image of electrospun polyamide- $6 / \mathrm{TiO}_{2}$ composite nanofibers.

In order to confirm the embedding of the nanoparticles into the nanofibers, we further performed HRTEM analysis. Figure 3 shows the HR-TEM images of electrospun polyamide- 6 composite nanofibers. The HR-TEM samples were obtained by placing the TEM grid very closely to the syringe micro-tip end for very short time during electrospinning. The as-spun polyamide- 6 nanofibers with the incorporation of $\mathrm{TiO}_{2}$ nanoparticles in polyamide- 6 nanofibers are clearly visible, as shown in Fig. 3(a). The crystal orientation in polyamide-6 nanofibers was further investigated by using selected area electron diffraction (SAED) pattern. Figure 3(b) presents a typical SAED pattern for a polymorphous phase, which shows that the $\mathrm{TiO}_{2}$ nanoparticles blended in polyamide- 6 nanofibers are of polymorphous nature. This consists of a series of fairly dotted concentric rings, which indicates that polyamide$6 / \mathrm{TiO}_{2}$ composite nanofibers contained a polycrystalline phase. From this result, it can be concluded that the polyamide- $6 / \mathrm{TiO}_{2}$ composite nanofibers contains both amorphous and polycrystalline phases.

The chemical composition of the as-spun nanofiber mats were analyzed by EDX spectrometer attached with TEM. Figure 4(a) and 4(b) shows the EDX spectrum of the polyamide- $6 / \mathrm{TiO}_{2}$ composite nanofibers. As shown in Fig. 4(b), the signals of oxygen and $\mathrm{Ti}$ were clearly observed for the composite nanofibers. The successful embedding of nanoparticles into polyamide- 6 nanofibers was confirmed by the EDX spectrum.

The luminescence properties of polyamide- $6 / \mathrm{TiO}_{2}$ are useful in understanding the physics of nanostructures and their potential applications in various fields. PL and CL spectra can also be used in order to study
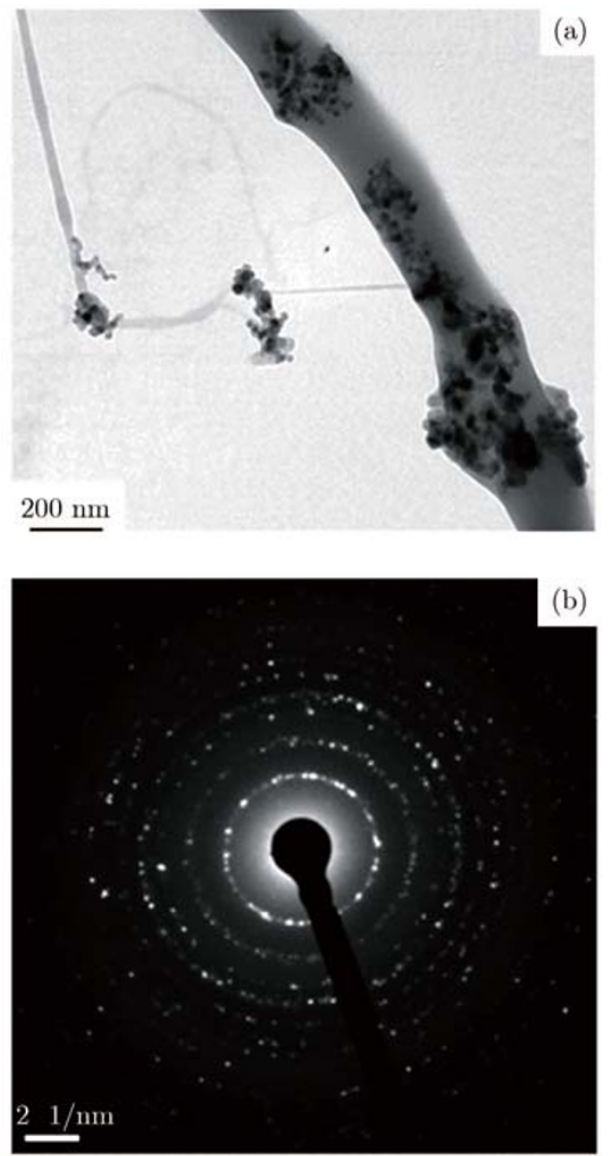

Fig. 3 HR-TEM image of electrospun polyamide- $6 / \mathrm{TiO}_{2}$ composite nanofibers and (b) its SAED pattern.

the polyamide- $6 / \mathrm{TiO}_{2}$ composite nanofibers energy levels and to provide information about the surface oxygen vacancies and defects based on the electronic structure and optical characteristics. Figure 5(a) shows the room-temperature PL spectrum of the electrospun polyamide- $6 / \mathrm{TiO}_{2}$ composite nanofibers. The $\mathrm{PL}$ spectrum of polyamide- $6 / \mathrm{TiO}_{2}$ is dominated by the broad band emission in the range of $350 \sim 600 \mathrm{~nm}$ with a maximum of about $475 \mathrm{~nm}$. A broad green photoluminescence peak in the visible range centered at about $550 \mathrm{~nm}$ was only detected in polyamide- $6 / \mathrm{TiO}_{2}$ composite nanofibers and could be attributed to the radiative recombination of self-trapped excitons localized within the surface defects of polyamide- $6 / \mathrm{TiO}_{2}$ composite nanofibers $[19,20]$. The broad band emission from the PL spectrum at room temperature indicates that the polyamide- $6 / \mathrm{TiO}_{2}$ nanofibers have good luminescence quality. The CL spectrum of electrospun polyamide- $6 / \mathrm{TiO}_{2}$ composite nanofibers was further investigated. The representative CL spectrum of electrospun polyamide- $6 / \mathrm{TiO}_{2}$ composite nanofibers is shown in Fig. 5(b), which has identical shape as the PL emission spectrum. The CL spectrum of the as spun polyamide- $6 / \mathrm{TiO}_{2}$ composite nanofibers shows an overlapping of two broad emission bands due to the con- 
tribution of polyamide- 6 (centered at about $475 \mathrm{~nm}$ ), which might originate from organic functional groups of polyamide- 6 and $\mathrm{TiO}_{2}$ nanoparticles (centered around $550 \mathrm{~nm})$.
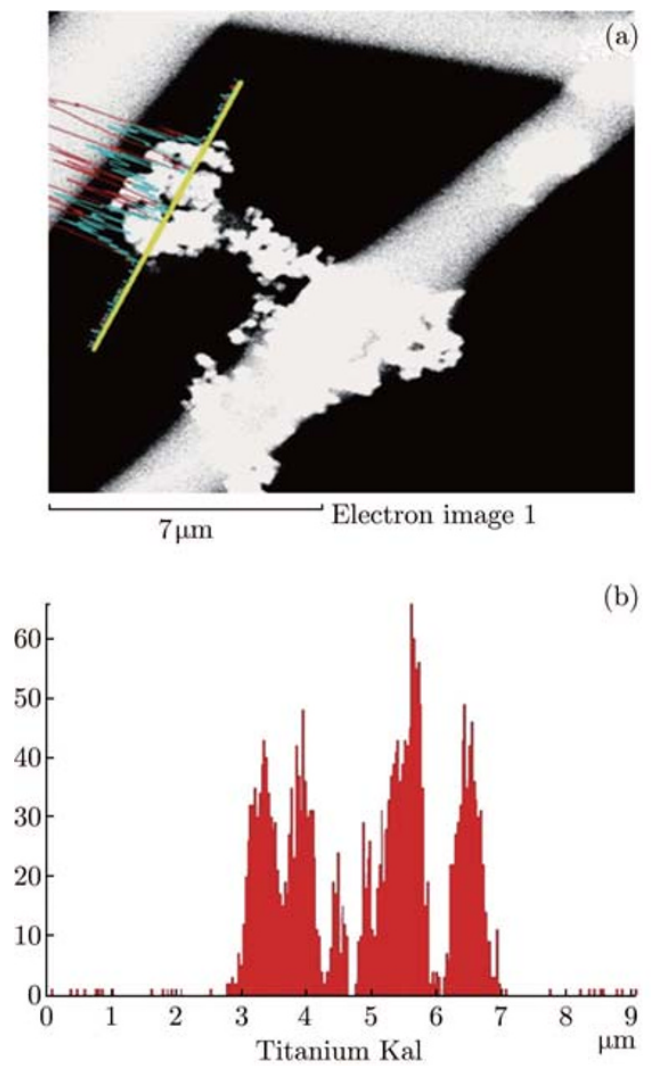

Fig. 4 (a) TEM-EDX spectrum of polyamide- $6 / \mathrm{TiO}_{2}$ composite nanofibers, showing the presence of $\mathrm{Ti}$ in the composite nanofibers (b).

Figures 6 8 show the electrical properties of the electrospun polyamide- $6 / \mathrm{TiO}_{2}$ composite nanofibers on silicon, paper and glass substrates, respectively. I-V dependence is typical for a metal-polymer-metal configuration, i.e., it is formed by the characteristics of two point-to-point contacts. The first evidence is the ohmic behavior of the composite nanofibers was due to the $\mathrm{I}-\mathrm{V}$ curves being linear. Figure 6 shows the I-V characteristics of electrospun polyamide- $6 / \mathrm{TiO}_{2}$ composite nanofibers on silicon substrate for the applied bias voltage in the range between -20 and $20 \mathrm{~V}$. The surface potential of the embedded nanoparticles were directly attributed to the enhanced electrical conduction in which field induced polarization was occurred due to applied bias voltage. The electrical conductivity of the polyamide- $6 / \mathrm{TiO}_{2}$ composite nanofibers on silicon substrate was of the order of $1 \sim 3 \mu \mathrm{A}$. However, this phenomenon may be attributed to the related contacts
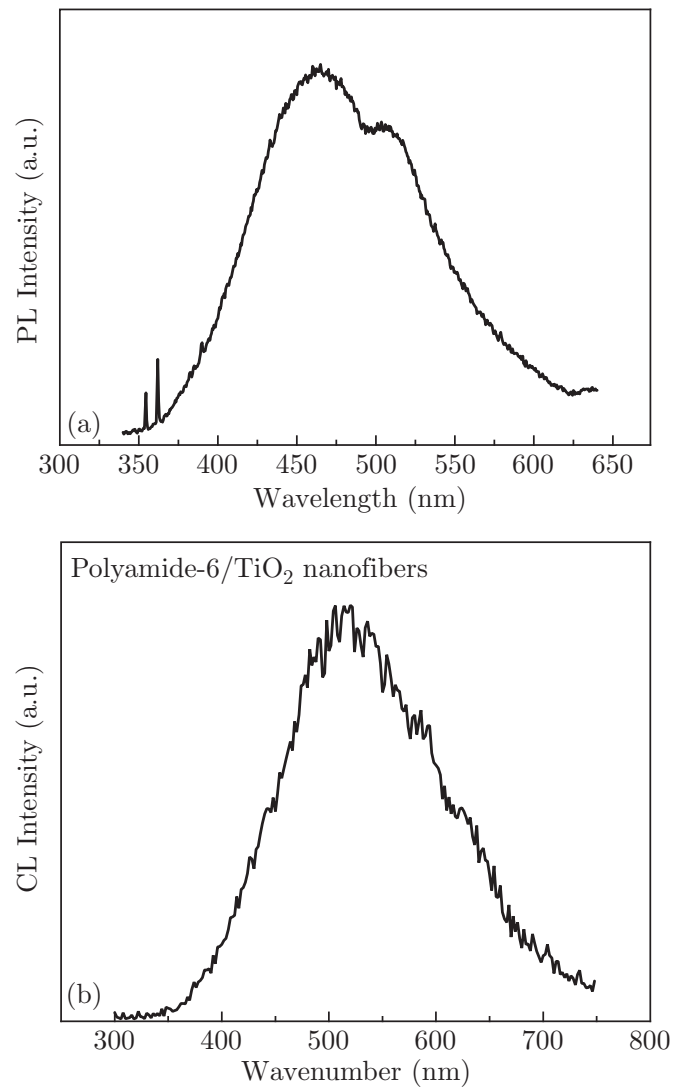

Fig. 5 (a) PL and (b) CL spectrum of electrospun polyamide- $6 / \mathrm{TiO}_{2}$ composite nanofibers.

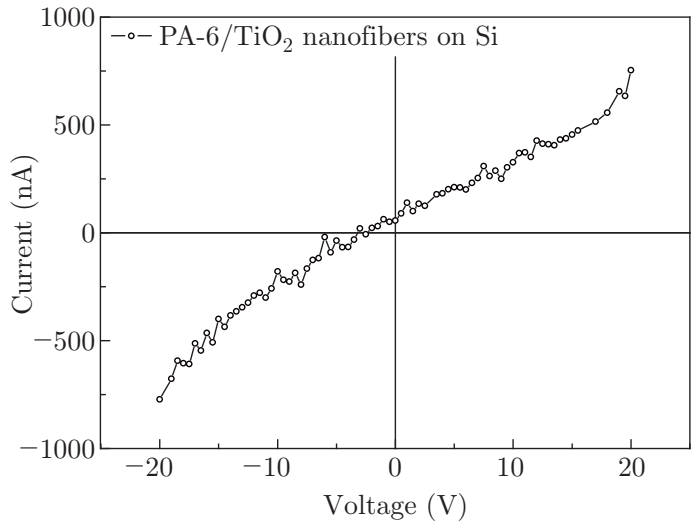

Fig. 6 I-V characteristics of electrospun polyamide- $6 / \mathrm{TiO}_{2}$ composite nanofibers on silicon substrate.

between the composite nanofibers, Ag electrodes, and $\mathrm{Si}$ substrate. Actually, we believe that the use of $\mathrm{Si}$ substrate when measuring the I-V properties of semiconductor nanostructures or polymers, the current may be leaking into the substrate. In order to understand the electrical transport of polyamide- $6 / \mathrm{TiO}_{2}$ composite nanofibers in a better way, we performed I-V characteristics on paper and glass substrates. The conductivity of the composite nanofibers on the paper and glass substrates were determined to be in the range of 1 to $20 \mathrm{pA}$ as shown in Figs. 7 and 8. The conductivity of the 
composite nanofibers was increased by about 6 orders of magnitude when compared to that of the paper and glass substrates. These observations indicate that the electrical conductivity of the polyamide- $6 / \mathrm{TiO}_{2}$ composite nanofibers can be significantly enhanced with the use of silicon substrates. The conductance measured on polyamide- $6 / \mathrm{TiO}_{2}$ composite nanofibers over different substrates was significantly affected. At the same time, we should also consider the electrical leakage occurred through the substrate when Si substrates were utilized $[21,22]$. In addition, in order to obtain good electrical characteristics with high stability, we should be further improved the interface and electrode contacts, which would extend the possibilities of high quality performance as per the device applications are concerned. In addition, we plan to optimize the experimental parameters so as to improve the electrical characteristics for device fabrication.

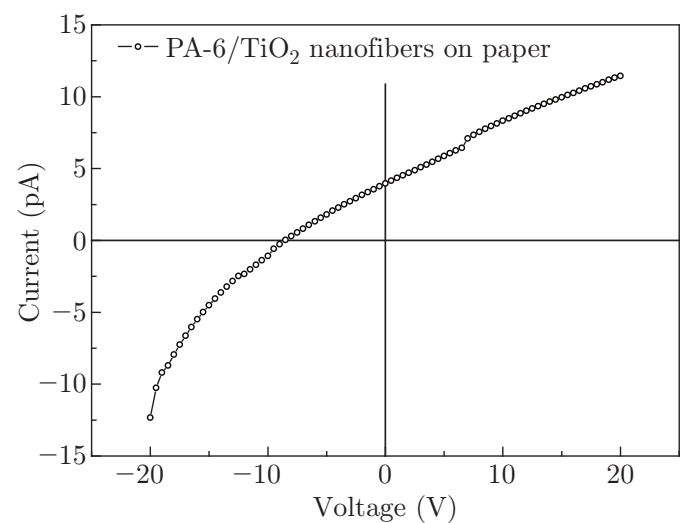

Fig. 7 I-V characteristics of electrospun polyamide- $6 / \mathrm{TiO}_{2}$ composite nanofibers on paper substrate.

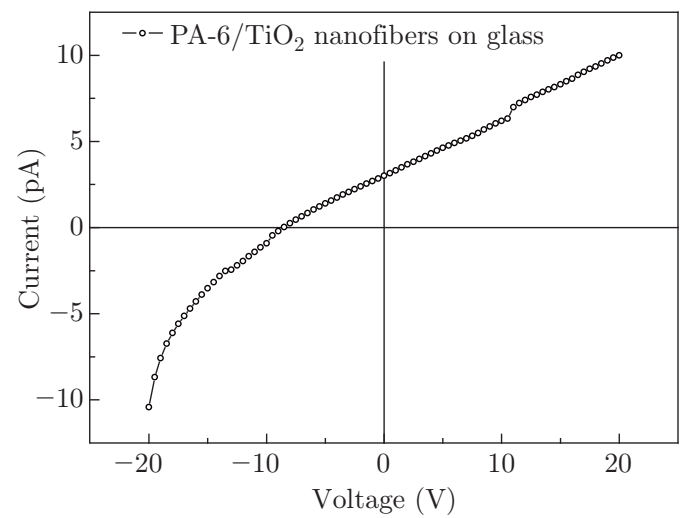

Fig. 8 I-V characteristics of electrospun polyamide- $6 / \mathrm{TiO}_{2}$ composite nanofibers on glass substrate.

On the basis of our experimental analysis, we conclude that the substrate play a significant role on the electrical properties of polyamide- $6 / \mathrm{TiO}_{2}$ composite nanofibers. It is worth noting that the improvement of electrical properties by addition of nanoparticles in the polyamide- 6 nanofibers is of key significance for the nano-device fabrication.

In summary, the electrical characteristics of polyamide-6 nanofibers embedded with $\mathrm{TiO}_{2}$ nanoparticles via electrospinning process have been investigated. The as-spun nanofibers were observed to be of smooth with uniform diameters along their lengths. The resultant composite nanofibers diameters were in the range of 100 to $150 \mathrm{~nm}$. The electrospinning process could be used to prepare polyamide- $6 / \mathrm{TiO}_{2}$ composite nanofibers with a well-defined morphology. The incorporation of $\mathrm{TiO}_{2}$ nanoparticles into the polyamide- 6 nanofibers were confirmed by HR-TEM and EDX analysis. The electrical characterization of the polyamide$6 / \mathrm{TiO}_{2}$ composite nanofibers showed a pure resistive behavior of the conductive nanofibers and a good stability of the as-spun nanostructures. The significantly enhanced electrical properties of these polyamide- 6 composite nanofibers can be utilized for promising nanotechnological applications. This observation will continue to drive researchers toward developing future organic based nano-devices.

\section{Acknowledgements}

This work was supported by a grant from the Korean Ministry of Education, Science and Technology (The Regional Core Research Program/Center for Healthcare Technology \& Development, Chonbuk National University, Jeonju 561-756 Republic of Korea).

\section{References}

[1] M. H. Huang, S. Mao, H. Feick, H. Q. Yan, Y. Y. Wu, H. Kind, E. Weber, R. Russo and P. D. Yang, Science 292, 1897 (2001). http://dx.doi.org/10.1126/ science. 1060367

[2] W. U. Huynh, J. J. Dittmer and A. P. Alivisatos, Science 295, 2425 (2002). http://dx.doi.org/10.1126/ science. 1069156

[3] R. Ramaseshan, S. Sundararajan and R. Jose, J. Appl. Phys. 102, 111101 (2007). http://dx.doi.org/ 10.1063/1.2815499

[4] Z. W. Pan, Z. R. Dai and Z. L. Wang, Science 291, 1947 (2001). http://dx.doi.org/10.1126/science. 1058120

[5] K. Tsukagoshi, B. W. Alphenaar and H. Ago, Nature 401, 572 (1999). http://dx.doi.org/10.1038/44108

[6] D. F. Zhang, L. D. Sun, C. J. Jia, Z. G. Yan, L. P. You and C. H. Yan, J. Am. Chem. Soc. 127, 13492 (2005). http://dx.doi.org/10.1021/ja054771k

[7] H. Zhang, H. W. Song and H. Q. Yu, J. Phys. Chem. C 111, 6524 (2007). http://dx.doi.org/10. 1021/jp0684123 
[8] H. Zhang, H. W. Song and H. Q. Yu, Appl. Phys. Lett. 90, 103103 (2007). http://dx.doi.org/10.1063/1. 2711380

[9] Y. C. Choi, W. S. Kim, Y. S. Park, S. M. Lee, D. J. Bae, Y. H. Lee, G. S. Park, W. B. Choi and N. S. Lee, J. M. Kim, Adv. Mater. 12, 746 (2000). http:// dx.doi.org/10.1002/(SICI) 1521-4095(200005) 12: $10 \$<\$ 746:$ : AID-ADMA746\$> $\$ 3.0 . \mathrm{CO} ; 2-\mathrm{N}$

[10] X. F. Duan and C. M. Lieber, Adv. Mater. 12, 298 (2000). http://dx.doi.org/10. 1002/(SICI) 1521-4095(200002) 12: 4\$<\$298: : AID-ADMA298\$>\$3. 0. CO; $2-Y$

[11] Y. Wu and P. Yang, Chem. Mater. 12, 605 (2000). http://dx.doi.org/10.1021/cm9907514

[12] Y. Li, G. W. Meng, L. D. Zhang and F. Phillip, Appl. Phys. Lett. 76, 2011 (2000). http://dx.doi.org/10. $1063 / 1.126238$

[13] R. Kessick and G. Tepper, Appl. Phys. Lett. 83, 557 (2003). http://dx.doi.org/10.1063/1.1594283

[14] J. Venugopal and S. Ramakrishna, Appl. Biochem. Biotech. 125, 147 (2005). http://dx.doi.org/10. 1385/ABAB : $125: 3: 147$

[15] R. Nirmala, R. Navamathavan, M. H. E. Newehy and H. Y. Kim, Mater. Lett. 65, 493 (2011). http://dx. doi.org/10.1016/j.matlet.2010.10.066
[16] B. Ding, M. Wang, J. Yu and G. Sun, Sensors 9, 1609 (2003). http://dx.doi.org/10.3390/s90301609

[17] Q. D. Ling, D. J. Liaw, C. Zhu, D. S. H. Chan, E. T. Kang and K. G. Neoh, Prog. Polym. Sci. 33, 917 (2008). http://dx.doi.org/10.1016/j. progpolymsci.2008.08.001

[18] J. Ananpattarachai, P. Kajitvichyanukul and S. Seraphin, J. Hazard. Mater. 168, 253 (2009). http:// dx.doi.org/10.1016/j.jhazmat.2009.02.036

[19] W. F. Zhang, M. S. Zhang, Z. Yin and Q. Chen, Appl. Phys. B: Lasers Opt. 70, 261 (2000). http://dx.doi. org/10.1007/s003400050043

[20] J. Liqiang, S. Xiaojun, C. Weimin, X. Zili, D. Yaoguo and F. Honggang, J. Phys. Chem. Solid 64, 615 (2003). http://dx.doi.org/10.1016/ S0022-3697 (02) 00362-1

[21] S. M. Kim, J. W. Son, K. R. Lee, H. Kim, H. R. Kim, H. W. Lee and J. H. Lee, J. Electroceram. 24, 153 (2010). http://dx.doi.org/10.1016/ s0022-3697 (02) 00362-1

[22] A. Cattaneo, L. Pirozzi, B. Morten and M. Prudenziati, Electrocomponent Sci. Technol. 6, 247 (1980). 